\title{
Analysing the potential of seq-to-seq models for incremental interpretation in task-oriented dialogue
}

\author{
Dieuwke Hupkes, Sanne Bouwmeester, and Raquel Fernández \\ Institute for Logic Language and Computation \\ University of Amsterdam \\ \{d.hupkes, raquel.fernandez\}@uva.nl \\ sanne.bouwmeesterlestudent.uva.nl
}

\begin{abstract}
We investigate how encoder-decoder models trained on a synthetic dataset of task-oriented dialogues process disfluencies, such as hesitations and self-corrections. We find that, contrary to earlier results, disfluencies have very little impact on the task success of seq-to-seq models with attention. Using visualisations and diagnostic classifiers, we analyse the representations that are incrementally built by the model, and discover that models develop little to no awareness of the structure of disfluencies. However, adding disfluencies to the data appears to help the model create clearer representations overall, as evidenced by the attention patterns the different models exhibit.
\end{abstract}

\section{Introduction}

The use of Recurrent Neural Networks (RNNs) to tackle sequential language tasks has become standard in natural language processing, after impressive accomplishments in speech recognition, machine translation, and entailment (e.g., Sutskever et al., 2014; Bahdanau et al., 2015b; Kalchbrenner et al., 2014). Recently, RNNs have also been exploited as tools to model dialogue systems. Inspired by neural machine translation, researchers such as Ritter et al. (2011) and Vinyals and Le (2015) pioneered an approach to opendomain chit-chat conversation based on sequenceto-sequence models (Sutskever et al., 2014). In this paper, we focus on task-oriented dialogue, where the conversation serves to fulfil an independent goal in a given domain. Current neural dialogue models for task-oriented dialogue tend to equip systems with external memory components (Bordes et al., 2017), since key information needs to be stored for potentially long time spans. One of our goals here is to analyse to what extent sequence-to-sequence models without external memory can deal with this challenge.
In addition, we consider language realisations that include disfluencies common in dialogue interaction, such as repetitions and self-corrections (e.g., I'd like to make a reservation for six, I mean, for eight people). Disfluencies have been investigated extensively in psycholinguistics, with a range of studies showing that they affect sentence processing in intricate ways (Levelt, 1983; Fox Tree, 1995; Bailey and Ferreira, 2003; Ferreira and Bailey, 2004; Lau and Ferreira, 2005; Brennan and Schober, 2001). Most computational work on disfluencies, however, has focused on detection rather than on disfluency processing and interpretation (e.g., Stolcke and Shriberg, 1996; Heeman and Allen, 1999; Zwarts et al., 2010; Qian and Liu, 2013; ?; ?). In contrast, our aim is to get a better understanding of how RNNs process disfluent utterances and to analyse the impact of such disfluencies on a downstream task-in this case, issuing an API request reflecting the preferences of the user in a task-oriented dialogue.

For our experiments, we use the synthetic dataset bAbI (Bordes et al., 2017) and a modified version of it called bAbI+ which includes disfluencies (Shalyminov et al., 2017). The dataset contains simple dialogues between a user and a system in the restaurant reservation domain, which terminate with the system issuing an API call that encodes the user's request. In bAbI+, disfluencies are probabilistically inserted into user turns, following distributions in human data. Thus, while the data is artificial and certainly simplistic, its goal-oriented nature offers a rare opportunity: by assessing whether the system issues the right API call, we can study, in a controlled way, whether and how the model builds up a relevant semantic/pragmatic interpretation when processing a disfluent utterance-a key aspect that would not be available with unannotated natural data. 


\section{Data}

In this section, we discuss the two datasets we use for our experiments: bAbI (Bordes et al., 2017) and bAbI+ (Shalyminov et al., 2017).

\section{1 bAbI}

The bAbI dataset consists of a series of synthetic dialogues in English, representing humancomputer interactions in the context of restaurant reservations. The data is broken down into six subtasks that individuate different abilities that dialogue systems should have to conduct a successful conversation with a human. We focus on Task 1, which tests the capacity of a system to ask the right questions and integrate the answers of the user to issue an API call that matches the user's preferences regarding four semantic slots: cuisine, location, price range, and party size. A sample dialogue can be found in example (4), Section 4.1.

Data The training data for Task 1 is deliberatively kept simple and small, consisting of 1000 dialogues with on average 5 user and 7 system utterances. An additional 1000 dialogues based on different user queries are available for validation and testing, respectively. The overall vocabulary contains 86 distinct words. There are 7 distinct system utterances and 300 possible API calls.

Baselines Together with the dataset, Bordes et al. (2017) present several baseline models for the task. All the methods proposed are retrieval based, i.e., the models are trained to select the best system response from a set of candidate responses (in contrast to the models we investigate in the present work, which are generative-see Section 3). The baseline models include classical information retrieval (IR) methods such as TFIDF and nearest neighbour approaches, as well as an end-to-end recurrent neural network. Bordes et al. demonstrate that the end-to-end recurrent architecture-a memory network (Sukhbaatar et al., 2015)—outperforms the classical IR methods as well as supervised embeddings, obtaining a $100 \%$ accuracy on retrieving the correct API calls.

\section{2 bAbI+}

Shalyminov et al. (2017) observe that the original bAbI data lack naturalness and variation common in actual dialogue interaction. To introduce such variation while keeping lexical variation constant, they insert speech disfluencies, using a fixed set of templates that are probabilistically applied to the user turns of the original bAbI Task 1 dataset. In particular, three types of disfluencies are introduced: hesitations (1), restarts (2), and selfcorrections (3), in around $21 \%, 40 \%$ and $5 \%$ of the user's turns, respectively. ${ }^{1}$

(1) We will be $u$ hm eight

(2) Good morning uhm yeah good morning

(3) I would like a French uhm sorry a Vietnamese restaurant

Eshghi et al. (2017) use the bAbI+ dataset to show that a grammar-based semantic parser specifically designed to process incremental dialogue phenomena is able to handle the bAbI+ data without having been directly exposed to it, achieving $100 \%$ accuracy on API-call prediction. They then investigate whether the memory network approach by Bordes et al. (2017) is able to generalise to the disfluent data, finding that the model obtains very poor accuracy (28\%) on API-call prediction when trained on the original bAbI dataset and tested on bAbI+. Shalyminov et al. (2017) further show that, even when the model is explicitly trained on bAbI+, its performance decreases significantly, achieving only $53 \%$ accuracy.

This result, together with the high level of control on types and frequency of disfluencies offered by the bAbI+ scripts, makes the bAbI+ data an excellent testbed for studying the processing of disfluencies by recurrent neural networks.

\section{Generative bAbI+ Modelling}

We start with replicating the results of Shalyminov et al. (2017) and Eshghi et al. (2017) using a generative rather than retrieval based model. For this replication, we use a vanilla one-layer encoderdecoder model (Sutskever et al., 2014) without any external memory. We train models with and without an attention mechanism (Bahdanau et al., 2015b) and compare their results. We perform a modest grid search over hidden layer and embedding sizes and find that an embedding size of 128 and a hidden layer size of 500 appear to be minimally required to achieve a good performance on the task. We therefore fix the embedding and hidden layer size to 128 and 500, respectively, for all further experiments.

\footnotetext{
${ }^{1}$ The inserted material is in italics in the examples.
} 


\begin{tabular}{|l||cc|cc||c|}
\hline \multicolumn{1}{|c||}{} & \multicolumn{2}{c|}{ seq2seq } & \multicolumn{2}{c|}{ attentive seq2seq } & MemN2N \\
\hline \multicolumn{1}{|c||}{ train / test } & utterances & API calls & utterances & API calls & API calls \\
\hline $\mathrm{bAbI} \mathrm{/} \mathrm{bAbI}$ & $100(100)$ & $0.02(66.4)$ & $100(100)$ & $100(100)$ & 100 \\
$\mathrm{bAbI}+$ / bAbI+ & $100(100)$ & $0.2(80.6)$ & $100(100)$ & $98.7(99.7)$ & 53 \\
$\mathrm{bAbI} / \mathrm{bAbI}+$ & $81.4(83.3)$ & $0.00(58.2)$ & $91.5(92.8)$ & $50.4(90.1)$ & 28 \\
$\mathrm{bAbI}+/ \mathrm{bAbI}$ & $100(100)$ & $0.2(81.4)$ & $100(100)$ & $99.2(100)$ & 99 \\
\hline
\end{tabular}

Table 1: Sequence accuracy (word accuracy in brackets) on the test set for utterances (non-API call responses) and API calls only. The last column shows accuracy on the test set for the retrieval-based memory-network system, as reported by Shalyminov et al. (2017) .

\subsection{Training}

All models are trained to predict the system utterances of all of the 1000 training dialogues of the $\mathrm{bAbI}$ and $\mathrm{bAbI}+$ dataset, respectively, including the final API call. After each user turn, models are asked to generate the next system utterance in the dialogue, given the dialogue history up to that point, which consists of all human and system utterances that previously occurred in that dialogue. The model's parameters are updated using stochastic gradient descent on a cross-entropy loss (using mini-batch size 32), with Adam (Kingma and $\mathrm{Ba}, 2014$ ) as optimiser (learning rate 0.001). All models are trained until convergence, which was reached after $\sim 20$ epochs.

\subsection{Evaluation}

Following Shalyminov et al. (2017), we use a $2 \times 2$ paradigm in which we train models either on $\mathrm{bAbI}$ or bAbI+ data and evaluate their performance on the test set of the same dataset, as well as across datasets. We report both the percentage of correct words in the generated responses (word accuracy) and the percentage of responses that were entirely correct (sequence accuracy). Additionally, we separately report the word and sequence accuracy of the API calls generated at the end of each dialogue. Note that these metrics are more challenging than the retrieval-based ones used by Bordes et al. (2017) and Eshghi et al. (2017), as the correct response has to be generated word by word, rather than merely being selected from a set of already available candidate utterances.

\subsection{Results}

Our results can be found in Table 1. The results obtained with the bAbI/bAbI and bAbI+/bAbI+ conditions indicate that an encoder-decoder model with attention can achieve near-perfect accuracy on Task 1 (predicting the right API call), whereas a model without attention cannot (sequence accuracy for API calls is only $0.02 \%$ on $\mathrm{bAbI} / \mathrm{bAbI}$ and $0.2 \%$ on bAbI+/bAbI+). This suggests that, in line with what was posed by Bordes et al. (2017), the bAbI Task 1 requires some form of memory that goes beyond what is available in a vanilla sequence-to-sequence model. To solve the task, however, using an attention mechanism sufficesa more complicated memory such as present in memory networks is not necessary.

Furthermore, our results confirm that models trained on data without disfluencies struggle to generalise when these are introduced at testing time (bAbI/bAbI+): While the overall accuracy of the dialogue is still high $(91.5 \%$ of utterances are correct), API call accuracy falls back to $50.4 \%$. Models trained on data containing disfluencies, however, show near-perfect accuracy on disfluent test data $(98.7 \%$ on bAbI+/bAbI+) - a result that stands in stark contrast with the findings of Eshghi et al. (2017) and Shalyminov et al. (2017).

\section{Generalisation to Disfluent Data}

In this section, we analyse the potential for generalisation of the encoder-decoder model with attention by focusing on the $\mathrm{bAbI} / \mathrm{bAbI}+$ condition, where the model trained on $\mathrm{bAbI}$ data is tested on bAbI+. As shown in Table 1, while the model performs perfectly on the bAbI corpus, it achieves only 50\% accuracy on API call prediction when it is asked to generalised to bAbI+ data. Here we aim to shed light on these results by studying the errors made by the model and visualising the patterns of the attention component of the network.

\subsection{Qualitative error analysis}

We start by observing that the model faced with the $\mathrm{bAbI} / \mathrm{bAbI}+$ condition encounters new lexical items at test time, such as filled pauses (uh) or editing terms (no sorry). These items are all 


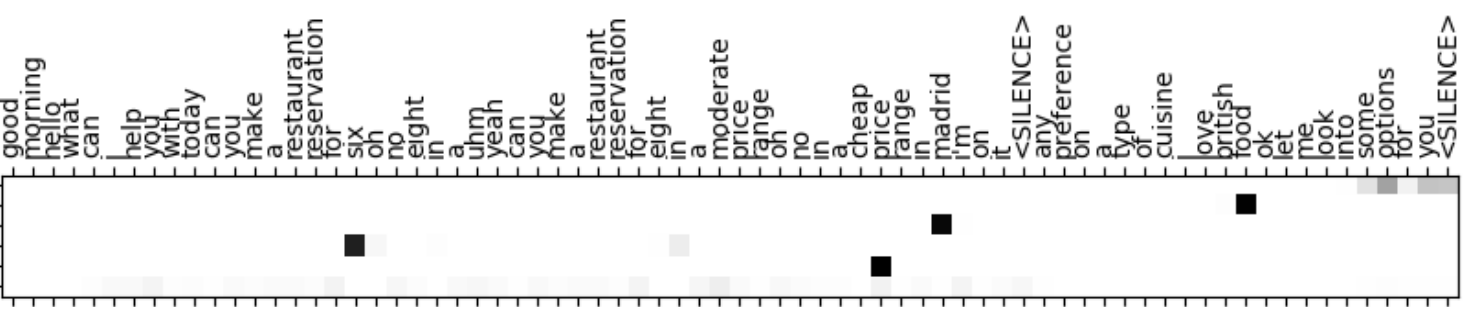

Figure 1: Visualisation of the decoder attention when generating the API call (vertical axis) for the disfluent dialogue in example (4) (horizontal axis). Darker colours indicate higher attention values.

mapped to a single token $<u n k>$ for 'unknown'. In addition, the presence of disfluencies increases the length of user utterances: The average utterance length in bAbI is 4.8 tokens, while user utterances in bAbI+ have an average length of 7.6.

Since the inventory of system utterances is very limited (there are 7 types of system outputs), the model has no difficulty learning their surface form. The errors we find, therefore, do not concern the grammaticality of the utterances, but rather when in the course of a dialogue a particular system utterance is offered. We find that utterance errors always involve a jump ahead in the expected sequence of system utterances. For example, around $83 \%$ of the utterance errors occur at the beginning of the dialogue. If the first user utterance is disfluent (e.g., Good morning <unk> <unk> good morning) and hence longer than expected, the model tends to skip the system's greeting and initial prompt (Hello, what can I help you with today) and instead outputs the second system prompt ( $I^{\prime} m$ on it), which only makes sense once the user has already provided some task-related information. Utterance errors of this kind, where the model generates an utterance that would be appropriate later on in the dialogue, occur after all disfluency types present in bAbI+: hesitations, restarts, and self-repairs.

The current evaluation setup does not allow us to test whether utterance errors by the system lead to errors in the final API call, since the input sequence given to the encoder is the gold dialogue history and not the dialogue history with system utterances generated by the model. This, however, allows us to conclude that the errors made by the model on around $50 \%$ of all API calls are a direct consequence of the disfluencies present in the user utterances. Not surprisingly, API call errors are triggered by self-corrections, which in bAbI+ always involve the mention of two different values for a single semantic slot (see example (4) be- low). Indeed, the model trained on bAbI achieves near-perfect accuracy when tested on a corpus that does not contain self-correction but only hesitations and restarts. This indicates that the lower accuracy is specifically caused by the presence of multiple potential values for the same API slot in self-corrections.

Manual error inspection shows that the model does not follow a systematic strategy whereby the first mention of a semantic slot is selected, which would lead to all self-corrections being processed incorrectly. As illustrated in (4), the model seems to randomly select between the first and the second mention of a slot in a self-correction structure, which leads to some self-corrections being processed correctly:

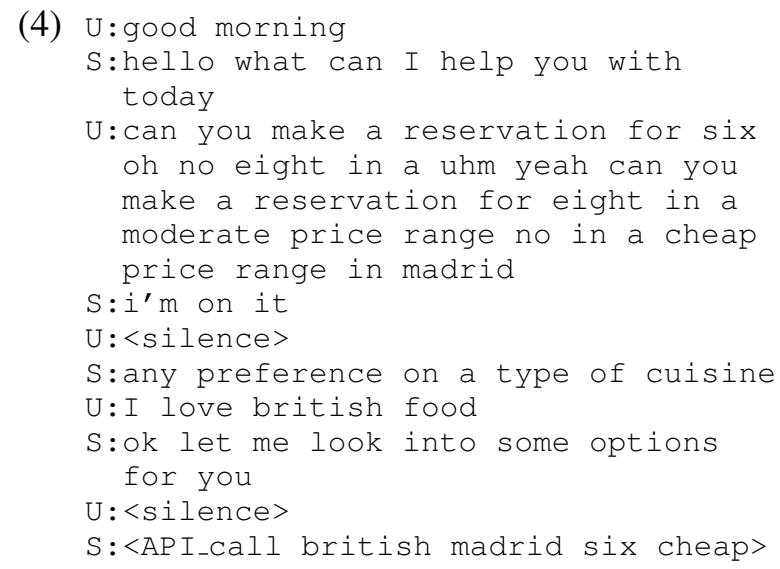

In Figure 1, we visualise the decoder attention of the bAbI model when it generates the (partly incorrect) API call for the dialogue in (4). The plot shows that while generating the different components of the API call, the model strongly relies on its attention mechanism to select the right slot. Furthermore, it confirms the observation that the model is not consistent in its strategy to select a slot after a disfluency: While it incorrectly attends to six (rather than eight), it correctly selects cheap (a repair of moderate). In the next section, we will have a closer look at the attention pat- 

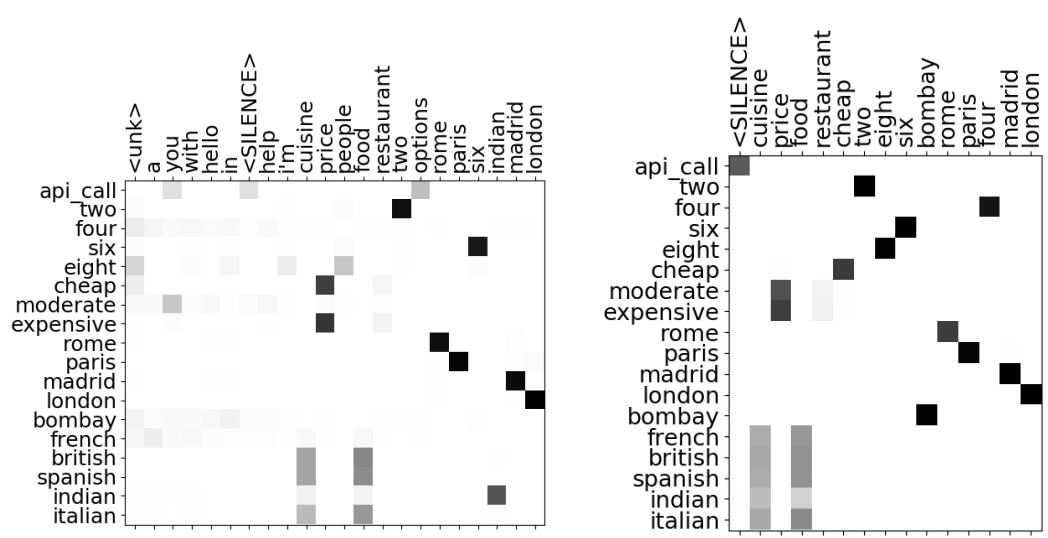

Figure 2: Alignment of in- and output words via the attention for different models tested on bAbI+ data. Left: model trained on bAbI. Right: model trained on bAbI+.

terns of both the bAbI and bAbI+ trained model.

\subsection{Comparing attention patterns}

To evaluate the network's attention patterns in a more quantitative way, we store all attention weights that the model computes while generating the API calls in the test corpus, and we compute their average for each API-call word. That is, for all words in an API-call, we compute to which words in the dialogue history the decoder was on average attending while it generated that word.

We plot the results in Figure 2, removing for each API-call word (on the vertical axis in the plot) the input words (horizontal axis) whose average attention score was lower 0.2 . We observe that the model trained on bAbI (left) not infrequently attends to $<$ unk $>$ while generating API calls, indicating that it did not attend to the correct content word. A striking difference appears comparing the results for the bAbI model with the bAbI+ trained model (Figure 2, right), whose attention scores are much less diffuse. While the bAbI model frequently attends to irrelevant words such as "hello", "a" or "in" (first columns in the plot), these words are not attended at all by the bAbI+ trained model. This difference suggests that the bAbI+ model developed a more clear distinction between different types of words in the input and benefits from the presence of disfluencies in the training data rather than being hindered by it.

In the next section, we investigate the representations developed by the bAbI+ model (bAbI+/bAbI+ condition), focussing in particular on how it incrementally processes disfluencies.

\section{Disfluency Processing}

In contrast to previous work (Eshghi et al., 2017; Shalyminov et al., 2017), our seq2seq model with attention trained on bAbI+ data learns to process disfluent user utterances remarkably well, achieving over $98 \%$ sequence accuracy on API calls (see bAbI+/bAbI+ condition in Table 1). In this section, we investigate how the model deals with disfluencies, in particular self-corrections, by drawing inspiration from human disfluency processing.

\subsection{The structure of disfluencies}

It has been often noted that disfluencies follow regular patterns (Levelt, 1983; Shriberg, 1994). Example (5) shows the structure of a prototypical self-correction, where the reparandum (RM) contains the problematic material to be repaired; the utterance is then interrupted, which can optionally be signalled with a filled paused and/or an editing term (ET); the final part is the repair (R) proper, after which the utterance may continue:

(5) a reservation for six $\{$ I mean $\}$ eight in a...

$$
\mathrm{RM} \text { ET } \mathrm{R}
$$

The presence or relationship between these elements serves to classify disfluencies into different types. For example, restarts such as those inserted in the bAbI+ corpus, are characterised by the fact that the reparandum and the repair components are identical (see example (2) in Section 2.2); in contrast to self-corrections, where the repair differs from and is intended to overwrite the material in the reparandum. In hesitations such as (1), there is only a filled pause and no reparandum nor repair. 


\subsection{Editing terms}

The algorithm used to generate the bAbI+ data systematically adds editing expressions (such as oh no or sorry) to all restarts and selfcorrections inserted in the data. However, editing expressions (e.g., I mean, rather, that is, sorry, oops) are in fact rare in naturally occurring human conversation. For example, Hough (2015) finds that only $18.52 \%$ of self-corrections in the Switchboard corpus contain an explicit editing term. Thus, while psycholinguistic research has shown that the presence of an explicit editing term followed by a correction makes the disfluency easier to handle (Brennan and Schober, 2001), humans are able to process disfluencies without the clues offered by such expressions.

Here we test whether the model relies on the systematic presence of editing expressions in the bAbI+ data. To this end, we created two new versions of the dataset using the code by Shalyminov et al. (2017): ${ }^{2}$ One with no editing term in any of the self-corrections or restarts, dubbed "noET"; and one where there is an editing term in $20 \%$ of self-corrections and restarts, dubbed "realET" as it reflects a more realistic presence of such expressions. We refer to the original bAbI+ data, which has editing terms in all self-corrections and restarts, as "fullET".

We test to what extent a model trained on fulIET, which could rely on the systematic presence of an editing term to detect the presence of a selfcorrection or restart, is able to process disfluencies in a more natural scenario where editing expressions are only sparsely available (realET). The result indicates that the editing term has very little effect on the model's performance: as shown in Table 2, accuracy goes down slightly, but is still extremely high (98\%). This finding persists when the editing terms are left out of the test data entirely (97\% accuracy when testing on noET). When models are trained on data containing fewer editing terms (realET and noET) and tested on data with a comparable or smaller percentage of editing terms, we observe a slightly larger drop in accuracy (see Table 2). We conclude that, although editing terms may help the model to develop better representations during training, their presence is not required to correctly process disfluencies at test time.

\footnotetext{
${ }^{2}$ https://github.com/ishalyminov/babi_ tools
}

\begin{tabular}{|c|c|c|c|}
\cline { 2 - 4 } \multicolumn{1}{c|}{} & \multicolumn{3}{c|}{ Tested on } \\
\hline Trained on & noET & realET & fullET \\
\hline fullET & 97 & 98 & 100 \\
\hline realET & 94 & 95 & \multicolumn{1}{c}{} \\
\hline noET & 94 & \multicolumn{2}{c}{} \\
\cline { 1 - 3 }
\end{tabular}

Table 2: Sequence accuracies of all sequences with and without editing term, averaged over 5 runs.

\subsection{Identification of structural components}

Disfluencies have regular patterns. However, identifying their components online is not trivial. The comprehender faces what Levelt (1983) calls the continuation problem: the need to identify (the beginning and end of the reparandum and the repair onset. Evidence shows that there are no clues (prosodic or otherwise) present during the reparandum. Thus the identification of the disfluency takes place at or after the moment of interruption (typically during the repair). Here there may be prosodic changes, but such clues are usually absent (Levelt and Cutler, 1983). Ferreira et al. (2004) point out that "the language comprehension system is able to identify a disfluency, likely through the use of a combination of cues (in some manner that is as yet not understood)."

We test to what extent our trained encoderdecoder model distinguishes reparanda and editing terms and can identify the boundaries of a repair using diagnostic classifiers (Hupkes et al., 2018). Diagnostic classifiers were proposed as a method to qualitatively evaluate whether specific information is encoded in high-dimensional representations-typically the hidden states that a trained neural network goes through while processing a sentence. The technique relies on training simple neural meta-models to predict the information of interest from these representations and then uses the accuracy of the resulting classifiers as a proxy for the extent to which this information is encoded in the representations.

In our case, we aim to identify whether the hidden layer activations reflect if the model is currently processing a reparandum, an editing term, or the repair. To test his, we label each word in the bAbI+ validation corpus according to which of the 3 categories it belongs to and train 3 binary classifiers to classify from the hidden layer activation of the encoder whether the word it just processed belongs to either one of these 3 classes. For an example of such a labelling we refer to Figure 3. 


$\begin{array}{lllllllllllllll}1 & 2 & 2 & 3 & 0 & 0 & 0 & 1 & 1 & 2 & 2 & 3 & 3 & 0 & 0\end{array}$

with uhm yeah with british cuisine in a moderate no sorry a cheap price range

Figure 3: A labelled example sentence to evaluate whether models have distinct representations for reparanda, repairs, and editing terms. For each label, we train a separate binary classifier to predict whether or not a word belongs to the corresponding class.

We hypothesise that while reparanda will not be detectable in the hidden layer activations, as they can only be identified as such a posteriori (Levelt, 1983; Ferreira et al., 2004), editing terms should be easy to detect, since they belong to a class of distinct words. The most interesting classifier we consider is the one identifying repairs, which requires a more structural understanding of the disfluency and the sentence as a whole.

\begin{tabular}{|l|l|c|}
\cline { 2 - 3 } \multicolumn{1}{c|}{} & self-corrections & restarts \\
\hline Reparandum & $15.0 / 89.4$ & 27.4 / 92.6 \\
Editing term & $37.3 / 99.4$ & $55.7 / 99.2$ \\
Repair & $21.3 / 93.5$ & $35.2 / 94.9$ \\
\hline
\end{tabular}

Table 3: Precision / recall of diagnostic classifiers to identify reparanda, editing terms and repairs.

The general trends in our results (see Table 3 above) are as expected: Editing terms are more easily recoverable than both reparanda and repairs, and the reparandum has the lowest scores with a precision and recall of 0.15 and 0.89 , respectively. However, results for editing terms and repairs are lower than expected. The presence of editing terms is not reliably identifiable given the hidden layer activations of a model $(37.3 \%$ and $55.7 \%$ precision for self-corrections and restarts, respectively), which is surprising given the fact that there is no overlap between editing terms and the rest of the model's vocabulary. Taken together with the results of our previous experiments in Section 5.2 regarding the effect of editing terms on the final sequence accuracy, this indicates that the presence of an editing term causes only minimal changes in the hidden layer activations, and thus leaves only a small trace in the hidden state of the network. The performance of the repair classifier is also low: $21.3 \%$ precision on self-correction and $35.2 \%$ on restarts. These results suggest that the model has no explicit representations of the structure of disfluencies and instead relies on other cues to infer the right API call.

\subsection{Incremental interpretation}

Next we analyse how the model processes disfluencies by looking into the interpretation-in terms of task-related predictions-that the model builds incrementally, word by word and utterance by utterance.

Word by word First, we probe the representations of the encoder part of the model while it processes incoming sentences, for which we use again diagnostic classifiers. In particular, we test if information that is given at a particular point in the dialogue (for instance, the user expresses she would like to eat Indian food) is remembered by the encoder throughout the rest of the conversation. We label the words in a dialogue according to what slot information was already provided previously in the dialogue, and test if this information can be predicted by a diagnostic classifier at later points in the dialogue. Note that while in the bAbI data the prediction for a slot changes only once when the user expresses her preference, due to the possibilities of corrections, slot information may change multiple times in the bAbI+ corpus. We train separate diagnostic classifiers for the different slots in the API call: cuisine (10 options), location (10 options), party size (4 options), and price range (3 options).

Our experiments show that the semantic information needed to issue an API call is not accurately predictable from the hidden representations that the encoder builds of the dialogue-see Table 4, where accuracy scores are all relatively low.

\begin{tabular}{|l|l|}
\hline Cuisine & 31.3 \\
Location & 25.9 \\
Price range & 57.3 \\
Party size & 43.0 \\
\hline
\end{tabular}

Table 4: Accuracy per slot type in the word-byword experiment.

In Figure 4, we plot the accuracy of the diagnostic classifiers over time, relative to the position at which information appears in the dialogue (that is, the accuracy at position 4 represents the accuracy 


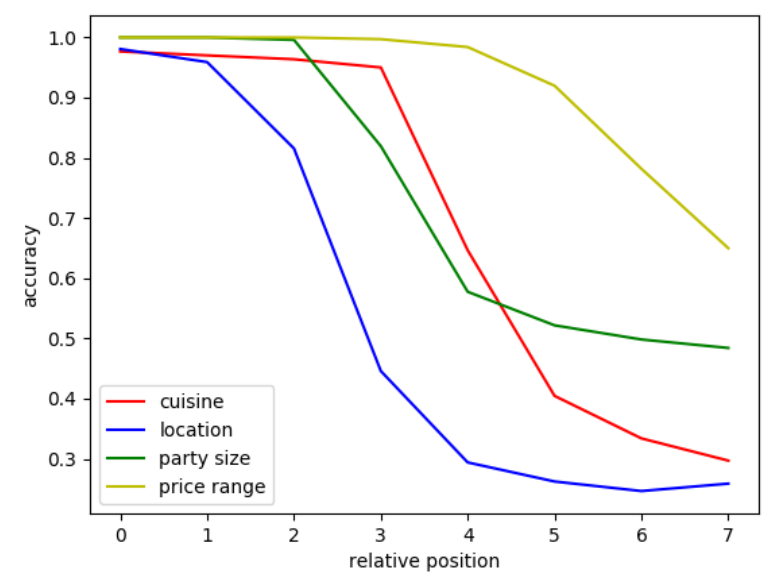

Figure 4: Accuracy at position relative to mention in the dialogue of each type of slot.

4 words after the slot information occurred). The plot illustrates that the encoder keeps traces of semantic slot information for a few time steps after this information appears in the dialogue, but then rapidly 'forgets' it when the dialogue continues. ${ }^{3}$ These results confirm our earlier findings that most of the burden for correctly issuing API calls falls on the model's attention mechanism, which needs to select the correct hidden states at the moment an API call should be generated.

Utterance by utterance In a second experiment, we study the incremental development of the API call made by the model's generative component (the decoder) by prompting it to generate an API call after every user utterance. To trigger the API calls, we append the utterances normally preceding an API call (let me look some options for you <silence>) to the dialogue history that is fed to the decoder. We apply this trick to elicit an API call after every user utterance in the dialogue. We evaluate the generated API calls by considering only the slots that can already be filled given the current dialogue history. That is, in a dialogue in which the user has requested to eat Italian food in London but has not talked about party size, we exclude the party size slot from the evaluation, and evaluate only whether the generated API call correctly predicts "Italian" and "London".

For models trained on bAbI data, the described method reliably prompted an API call, while it

\footnotetext{
${ }^{3}$ To exclude the possibility that the low accuracy is a consequence of relocation of information instead of it being forgotten, we also trained diagnostic classifiers to only start predicting a few words after slot information appears, but this did not result in an increase in accuracy.
}

\begin{tabular}{|l|c|}
\hline bAbI / bAbI & 100 \\
bAbI+ / bAbI & 100 \\
\hline bAbI / bAbI+ & 66.6 \\
bAbI+ / bAbI+ & 99.8 \\
\hline
\end{tabular}

Table 5: Accuracy on triggered API calls utterance by utterance.

was less successful for models trained on bAbI+, where API calls were evoked only in $86 \%$ of the time (when testing on bAbI+ data) and $54 \%$ of the time (when testing on bAbI data). For our analysis, we consider only cases in which the API call was prompted and ignore cases in which other sentences were generated

As shown in Table 5, we find that the decoders of both the bAbI and bAbI+ models are able to generate appropriate API calls immediately after slots are mentioned in the user utterance ( $\sim 100 \%$ accuracy in the bAbI/bAbI, bAbI+/bAbI, and $\mathrm{bAbI}+/ \mathrm{bAbI}+$ conditions). However, when confronted with disfluencies, the model trained on the disfluency-free bAbI data is not able to do so reliably (66.6\% accuracy with bAbI/bAbI+), following the trend we also observed in Table 1.

\section{Conclusions}

We have investigated how recurrent neural networks trained in a synthetic dataset of taskoriented English dialogues process disfluencies. Our first conclusion is that, contrary to earlier findings, recurrent networks with attention can learn to correctly process disfluencies, provided they were presented to them at training time. In the current data, they do so without strongly relying on the presence of editing terms or identifying the repair component of disfluent structures. When comparing models trained on data with and without disfluencies, we observe that the attention patterns of the former models are more clear-cut, suggesting that the disfluencies contribute to a better understanding of the input, rather than hindering it.

Furthermore, we find that in an encoder-decoder model with attention, at least for the current taskoriented setting, a large burden of the processing falls on the generative part of the model: the decoder aided by the attention mechanism. The encoder, on the other hand, does not incrementally develop complex representations of the dialogue history, limiting its usefulness as a cognitive model of language interpretation. We preliminary conclude that different learning biases are neces- 
sary to obtain a more balanced division of labour between encoder and decoder.

Here we have exploited synthetic data, taking advantage of the control this affords regarding types and frequency of disfluency patterns, as well as the direct connection between language processing and task success present in the dataset. In the future, we aim at investigating neural models of disfluency processing applied to more naturalistic data, possibly leveraging eye-tracking information to ground language comprehension (??).

\section{References}

Dzmitry Bahdanau, Kyunghyun Cho, and Yoshua Bengio. 2015a. Neural machine translation by jointly learning to align and translate. In Proceedings of the 3rd International Conference on Learning Representations (ICLR).

Dzmitry Bahdanau, Kyunghyun Cho, and Yoshua Bengio. 2015b. Neural machine translation by jointly learning to align and translate. In Proceedings of the 3rd International Conference on Learning Representations (ICLR2015).

Karl GD Bailey and Fernanda Ferreira. 2003. Disfluencies affect the parsing of garden-path sentences. Journal of Memory and Language, 49(2):183-200.

Antoine Bordes, Y-Lan Boureau, and Jason Weston. 2017. Learning end-to-end goal-oriented dialog. In Proceedings of the 5th International Conference on Learning Representations (ICLR).

Susan E Brennan and Michael F Schober. 2001. How listeners compensate for disfluencies in spontaneous speech. Journal of Memory and Language, 44(2):274-296.

Arash Eshghi, Igor Shalyminov, and Oliver Lemon. 2017. Bootstrapping incremental dialogue systems from minimal data: the generalisation power of dialogue grammars. In Proceedings of the 2017 Conference on Empirical Methods in Natural Language Processing (EMNLP), pages 2220-2230. Association for Computational Linguistics.

Fernanda Ferreira and Karl G. D. Bailey. 2004. Disfluencies and human language comprehension. Trends in Cognitive Sciences, 8(5).

Fernanda Ferreira, Ellen F. Lau, and Karl G. D. Bailey. 2004. Disfluencies, language comprehension, and tree adjoining grammars. Cognitive Science, 28:721-749.

Jean E. Fox Tree. 1995. The effects of false starts and repetitions on the processing of subsequent words in spontaneous speech. Journal of memory and language, 34(6):709-738.
Peter A. Heeman and James F. Allen. 1999. Speech repairs, intonational phrases, and discourse markers: modeling speakers' utterances in spoken dialogue. Computational Linguistics, 25(4):527-571.

Julian Hough. 2015. Modelling Incremental SelfRepair Processing in Dialogue. Ph.D. thesis, Queen Mary University of London.

Dieuwke Hupkes, Sara Veldhoen, and Willem Zuidema. 2018. Visualisation and 'diagnostic classifiers' reveal how recurrent and recursive neural networks process hierarchical structure. Journal of Artificial Intelligence Research, 61:907-926.

Nal Kalchbrenner, Edward Grefenstette, and Phil Blunsom. 2014. A convolutional neural network for modelling sentences. In Proceedings of the 52nd Annual Meeting of the Association for Computational Linguistics, pages 655-665.

Diederik Kingma and Jimmy Ba. 2014. Adam: A method for stochastic optimization. In Proceedings of ICLR 2014, pages 1-13.

Ellen F. Lau and Fernanda Ferreira. 2005. Lingering effects of disfluent material on comprehension of garden path sentences. Language and Cognitive Processes, 20:633-666.

Willem J. M. Levelt. 1983. Monitoring and self-repair in speech. Cognition, 14(1):41-104.

Willem JM Levelt and Anne Cutler. 1983. Prosodic marking in speech repair. Journal of semantics, 2(2):205-218.

Xian Qian and Yang Liu. 2013. Disfluency detection using multi-step stacked learning. In Proceedings of the 2013 Conference of the North American Chapter of the Association for Computational Linguistics: Human Language Technologies, pages 820-825.

Alan Ritter, Colin Cherry, and William B. Dolan. 2011. Data-driven response generation in social media. In Proceedings of the 2011 Conference on Empirical Methods in Natural Language Processing, pages 583-593. Association for Computational Linguistics.

Igor Shalyminov, Arash Eshghi, and Oliver Lemon. 2017. Challenging neural dialogue models with natural data: Memory networks fail on incremental phenomena. In Proceedings of the 21st Workshop on the Semantics and Pragmatics of Dialogue (SEMDIAL), pages 125-133.

Elizabeth E. Shriberg. 1994. Preliminaries to a Theory of Speech Disfluencies. Ph.D. thesis, University of California at Berkeley, Berkeley, CA.

Andreas Stolcke and Elizabeth Shriberg. 1996. Statistical language modeling for speech disfluencies. In Conference Proceedings of the IEEE International Conference on Acoustics, Speech, and Signal Processing (ICASSP-96), volume 1, pages 405-408. 
Sainbayar Sukhbaatar, arthur szlam, Jason Weston, and Rob Fergus. 2015. End-to-end memory networks. In C. Cortes, N. D. Lawrence, D. D. Lee, M. Sugiyama, and R. Garnett, editors, Advances in Neural Information Processing Systems 28, pages 2440-2448. Curran Associates, Inc.

Ilya Sutskever, Oriol Vinyals, and Quoc V Le. 2014. Sequence to sequence learning with neural networks. In Advances in neural information processing systems (NIPS), pages 3104-3112.

Oriol Vinyals and Quoc V. Le. 2015. A neural conversational model. In Proceedings of the 31 st International Conference on Machine Learning (ICML) Deep Learning Workshop.

Simon Zwarts, Mark Johnson, and Robert Dale. 2010. Detecting speech repairs incrementally using a noisy channel approach. In Proceedings of the 23rd international conference on computational linguistics, pages 1371-1378. Association for Computational Linguistics. 